\title{
Helminthic Infections of the Liver
}

\author{
Paul J. Pockros, MD* and Thomas A. Capozza, MD
}

\begin{abstract}
Address
*Division of Gastroenterology/Hepatology, Liver Research Consortium, Scripps Clinic, 10666 North Torrey Pines Road, La Jolla,

CA 92037, USA.

E-mail: ppockros@scrippsclinic.com

Current Gastroenterology Reports 2004, 6:287-296

Current Science Inc. ISSN I522-8037

Copyright () 2004 by Current Science Inc.

Human helminthic infestation is exceedingly common on a global scale, with as many as 1.5 to 2 billion people affected worldwide. Helminths (parasitic worms) that infect the liver and hepatobiliary system include nematodes (roundworms), cestodes (tapeworms), and trematodes (flatworms or flukes). The majority of morbidity and mortality from these infestations is caused by the host immune response to the larvae or adult worm. Helminthic disease manifestations vary from the extremes of asymptomatic carriage to cirrhosis and decompensated liver disease. Current basic science and clinical research focus on improvements in medical therapy, mass screening and chemoprophylaxis, and the development of preventative vaccine strategies.
\end{abstract}

\section{Introduction}

One of the most fascinating and appalling discoveries made by every student of nature and medicine is the close and frequent association between humans and parasitic worms. In fact, the recognition of this association dates back to ancient Mesopotamia, where Ascaris is the earliest recorded human helminthic infestation. Today, it is likely that as many as 1.5 to 2 billion people worldwide have been infested with helminths. Current research focuses largely on improved diagnostic testing and treatment, as well as understanding of the host-parasite immune response and harnessing that information for the development of better preventive strategies.

\section{Nematodes (Roundworms) Ascaris lumbricoides \\ Background}

Ascaris lumbricoides is the largest intestinal nematode of humans, measuring 30 to $50 \mathrm{~cm}$ in length and 3 to $6 \mathrm{~mm}$ in diameter (Fig. 1). It is estimated that 1 to 1.4 billion people worldwide are affected by ascariasis, making it the most common helminthic infection in humans $[1,2 \bullet \bullet]$. Its prevalence is highest in countries with warm, moist climates and poor sanitation. The majority of cases are seen in Asia (72\%), Africa (12\%), and South America (8\%). In several regions of southeast Asia, prevalence rates are as high as $92 \%$ in children $[3,4]$. In the United States, ascariasis is the third most common helminthic infection with approximately 4 million people affected; the majority of cases arise in immigrants from endemic regions [5].

\section{Mechanism of disease}

Infestation begins with the ingestion of embryonated $A$. lumbricoides eggs. In children, the mode of transmission is typically soil-contaminated hands, fingers, toys, and food. The larvae hatch in the duodenum, penetrate the mucosa of the small bowel, and enter either the portal circulation or small bowel lymphatics. The larvae then migrate to the liver sinusoids or the lungs. The larvae penetrate the alveoli, migrate up the tracheobronchial tract, and are swallowed by the host. After reaching the small bowel, the larvae mature into the adult form over 2 to 3 months. The average life span of an adult worm is 10 to 24 months. Fertilized eggs are passed in the host feces, become infectious, and remain viable in the soil for up to 10 years.

The pathogenesis of disease can be attributed to the following factors: 1) direct tissue damage from the migrating larvae; 2) sequelae of the host immune response to larvae; 3 ) physical obstruction of the intestine or hepatobiliary tract; and 4) nutritional deficiencies caused by a large worm burden [6].

\section{Clinical findings}

The vast majority of infected individuals are asymptomatic. Hepatobiliary and pancreatic disease is most commonly caused by direct mechanical obstruction of the pancreatobiliary system from the adult worm migrating across the ampulla of Vater. As a result, patients infected with Ascaris can present with biliary colic (56\%), tender hepatomegaly (50\%), acute cholangitis (24\%), acalculous cholecystitis (13\%), acute pancreatitis (6\%), and occasionally hepatic abscess [7]. In addition, dead worms or ova may serve as a nidus for the formation of pigment stones, which can lead to recurrent pyogenic cholangitis after the active infection has been treated successfully [8]. Patients with longstanding, untreated recurrent pyogenic cholangitis are at risk for developing secondary biliary cirrhosis. A case of granulomatous hepatitis due to Ascaris ova has also been reported [9]. 


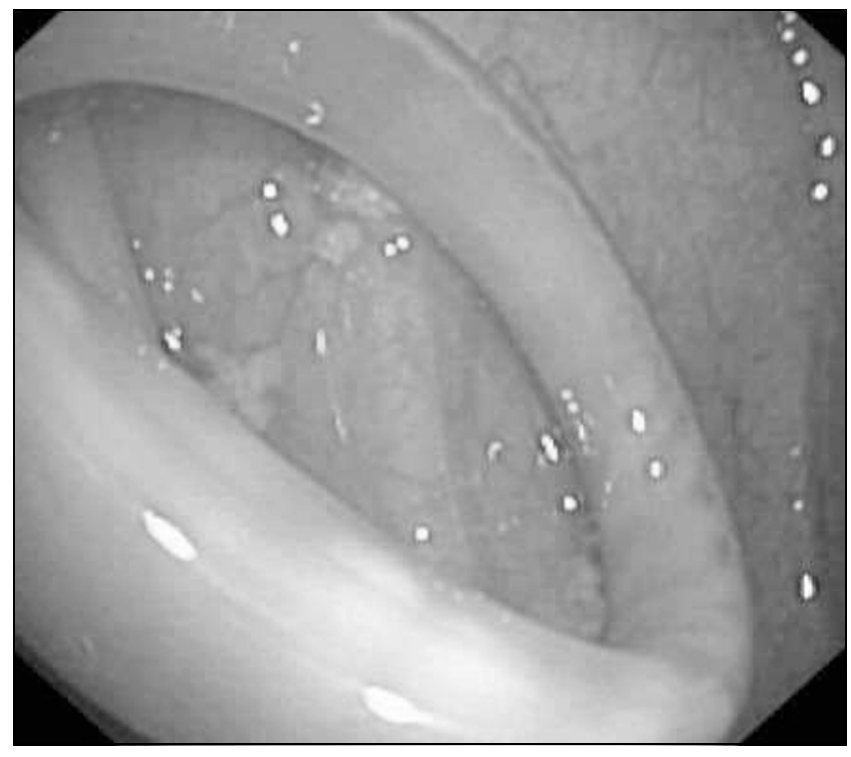

Figure 1. Ascaris lumbricoides, $24 \mathrm{~cm}$ long, in the intestine of a patient from the Phillipines. (Courtesy of Naval Medical Center, San Diego.)

\section{Diagnosis}

The diagnosis of ascariasis is made with the identification of an adult worm, larvae, or egg from a patient. Ascaris eggs can be detected in feces 40 to 60 days after exposure (if the patient is infected solely with male worms no eggs will be present in the feces). Hyperbilirubinemia is seen in $10 \%$ to $20 \%$ of cases. Mild to moderate peripheral eosinophilia (400-3000 eosinophils $/ \mathrm{cm}^{3}$ of blood) is associated with the larval stages of Ascaris infection but is absent during the intestinal infestation with adult worms [10•].

Transabdominal ultrasound, CT scan, or magnetic resonance imaging (MRI) can be useful in diagnosing hepatobiliary ascariasis. Ultrasound has the advantage of being readily available, inexpensive, and capable of real-time imaging; the detection of long, linear acoustic shadows within the biliary system coupled with movement on prolonged imaging is virtually diagnostic of biliary ascariasis. In a large retrospective review, ultrasonography correctly diagnosed the presence of worms in 52\% of patients [11]. Endoscopic retrograde cholangiopancreatography (ERCP) has also been used successfully to aid in the diagnosis of difficult cases.

\section{Therapeutic strategy}

Endoscopic retrograde cholangiopancreatography is the primary therapeutic modality in biliary obstruction caused by Ascaris infestation. Endoscopic extraction can be performed with forceps if the adult worm is protruding from the papilla. If the worm is within the common bile duct (or higher in the hepatobiliary tract) a dormia basket can be used to pull it down into the duodenum. Complete clearance can be obtained in the majority of patients and is strongly recommended to avoid leaving a nidus for future stone formation [11].
All patients diagnosed with Ascaris infection should receive antihelminthic therapy. The current treatment of choice is with one of two benzimidazole compounds: albendazole or mebendazole. Single-dose therapy with albendazole, $400 \mathrm{mg}$, is effective in more than $97 \%$ of cases [12]. Mebendazole (500 mg as single dose or $100 \mathrm{mg}$ twice daily for 3 days) is nearly as efficacious and available in a less expensive generic formulation but with a higher incidence of gastrointestinal side effects. Piperazine citrate has been recommended in pregnancy because of concerns about teratogenicity with benzimidazoles. Ivermectin is a highly effective single-dose antihelminthic agent with excellent coverage of intestinal nematodes; however, it is not approved by the US Food and Drug Administration (FDA) for the treatment of Ascaris infection [13].

\section{Strongyloides stercoralis \\ Background}

Strongyloides stercoralis is found most commonly in tropical and subtropical regions of the world but can also be found in the southeastern United States. An estimated 50 to 100 million people worldwide are infected with $S$. stercoralis $[2 \bullet \bullet]$. Strongyloides organisms are able to reproduce and complete their life cycle entirely within the host (autoinfection). Due to low-grade autoinfection, intestinal strongyloidiasis has been reported to persist for up to 40 years $[2 \bullet \bullet]$. This is an especially important consideration when caring for immigrants from endemic regions, liver transplant patients, or military veterans who may have served in endemic areas of the world.

\section{Mechanism of disease}

Infestation begins when the filariform larvae from contaminated soil penetrate the human skin or mucous membranes and enter the venous blood supply. The larvae are then carried to the lungs, cross the alveolar membrane, migrate up the tracheobronchial tract, and are swallowed. Once the larvae reach the duodenum and jejunum they mature into adult worms. The female adult burrows into the small bowel mucosa and begins depositing eggs, which are shed in the host feces. Adult worms have been reported to survive for up to 5 years in the human intestine. Autoinfection occurs when filariform larvae penetrate the colonic mucosa or perianal skin, thus completing the life cycle.

The pathogenesis of hepatic disease in strongyloidiasis is from overwhelming infestation (hyperinfection syndrome). Massive numbers of filariform larvae can invade the liver directly, resulting in a severe granulomatous reac-

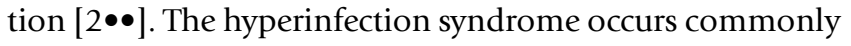
in immune-compromised individuals and therefore appears directly related to decreases in cellular immunity [14]. Notable risk factors in endemic regions include HIV and human T-cell leukemia/lymphoma virus infection, alcoholism and alcoholic cirrhosis [15], malignancy, chemotherapy, corticosteroid use, and malnutrition states. Recently, a case of hyperinfection syndrome was reported 
during therapy for hepatic $\mathrm{C}$ virus infection with interferon and ribavirin [16•]. Serum IgA and IgE have been reported to play a central role in suppressing larval output and autoinfection [17].

\section{Clinical findings}

Approximately one third of patients infected with Strongyloides are asymptomatic. Filariform larvae can produce a serpiginous urticarial rash due to their cutaneous migration (termed larva currens). Initial laboratory evaluation may reveal a mild to moderate peripheral eosinophilia [10•]. Eosinophilia may be absent in overwhelming infection or with corticosteroid use. In a recent review from Taiwan, $30 \%$ of patients chronically infected with Strongyloides had chronic liver disease or cirrhosis [18].

In the hyperinfection syndrome, the clinical presentation is more dramatic, with respiratory failure, profound diarrhea, electrolyte disturbance, malabsorption, and sepsis. Patients may become jaundiced and develop hepatomegaly caused by intrahepatic cholestasis. The overall mortality rate is approximately $25 \%$ in chronic strongyloidiasis, but it can be as high as $50 \%$ in patients with hyperinfection syndrome [18].

\section{Diagnosis}

Larvae are detectable in the stool or small bowel aspirate approximately 3 to 4 weeks after initial infection. However, up to $25 \%$ of patients may have negative stool studies [19]. Duodenal aspirates or mucosal biopsies can be helpful if stool studies are negative. The string test can also be used to provide samples from the duodenum to aid in diagnosis. Highly sensitive and specific immunofluorescence and enzyme-linked immunosorbent assays (ELISA) are available. These tests are most helpful in chronically infected or immunocompromised patients in conjunction with stool studies [20].

In disseminated disease, the larvae can be detected in most body fluids, including sputum, ascites, and pleural fluid. On liver biopsy, a mixed portal infiltrate of eosinophils, plasma cells, and macrophages, as well as a moderate lobular steatosis, may be seen. The filariform larvae can also be found in the bile ducts and in the hepatic parenchyma.

\section{Therapeutic strategy}

Ivermectin is the drug of choice for Strongyloides infestation $[13,21 \bullet \bullet$. Ivermectin, $200 \mu \mathrm{g} / \mathrm{kg} / \mathrm{d}$ for 1 to 2 days, is effective in up to $83 \%$ of patients with chronic strongyloidiasis. Thiabendazole, an older benzimidazole, is recommended as an alternative therapy and may be the preferred drug in hyperinfection syndrome. Thiabendazole is given orally, 50 $\mathrm{mg} / \mathrm{kg}$, in two doses for 2 days; however, the dosage may

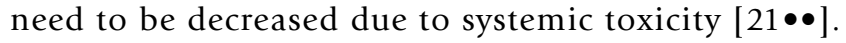
Albendazole, $400 \mathrm{mg}$ twice daily for 3 to 10 days, has been suggested as a more tolerable alternative to thiabendazole as second-line therapy for Strongyloides infection [22].

\section{Toxocara canis}

\section{Background}

The dog roundworm Toxocara canis is distributed worldwide and found in close association with domesticated pets. It is one of the most common human helminthic infections in developed countries, with a serologic prevalence reported at $2 \%$ in the United States [23]. T. canis infection is seen mainly in children aged under 5 years.

\section{Mechanism of disease}

Toxocara infestation begins with the ingestion of embryonated eggs from contaminated soil. Larvae hatch in the small intestine, penetrate the mucosa, and are delivered to the liver via the portal circulation. Once the larvae reach the capillary beds of the various organs, they penetrate the vascular wall and migrate into the surrounding host tissue. This passage into the viscera and the resultant host immune response give rise to toxocariasis or visceral larva migrans. As with other helminths, the larvae travel to the lungs, are aspirated, and mature into adult worms in the small intestine of their definitive host.

The larval invasion into the surrounding tissue incites an inflammatory response characterized by eosinophilic granulomas as well as necrosis and hemorrhage [24]. The larvae can survive for a prolonged period of time despite the vigorous immune response and in later stages cause fibrosis and calcification [24].

\section{Clinical findings}

The majority of Toxocara infections are mild and asymptomatic except for the presence of a moderate peripheral eosinophilia [23]. In heavier infestation, the early stage of disease can include fever, malaise, anorexia, marked eosinophilia, respiratory difficulties, and hepatomegaly. As the visceral migration occurs, symptoms are specific to each organ system, including pulmonary infiltrates, encephalitis, seizures, myocarditis, endophthalmitis, and retinal granulomas. Patients can develop granulomatous hepatitis, hepatic abscesses, and/or tender hepatosplenomegaly [23].

\section{Diagnosis}

The gold standard for the diagnosis of toxocariasis is detection of larvae on tissue biopsy. Hypergammaglobulinemia is also common, with elevated levels of IgG, IgM, and IgE $[2 \bullet \bullet, 23]$. An ELISA test for Toxocara species is available for confirmation and is considered the most accurate method of diagnosis $[23,24]$. A polymerase chain reaction (PCR)based method has been used in evaluating liver biopsy specimens [25]. On liver histology, a mixed portal infiltrate, hepatic granulomas, or small eosinophilic abscesses can be seen [14]. Stool studies are of no benefit because Toxocara species do not complete their life cycle in humans. Ultrasonography of the liver reveals hypoechoic lesions, and MRI shows ill-defined areas of necrosis with surrounding edema in the hepatic parenchyma $[23,24]$. 


\section{Therapeutic strategy}

The majority of cases are self-limited and require no antihelminthic therapy. In severe cases of visceral larva migrans, albendazole, $400 \mathrm{mg}$ twice daily for 5 days (or mebendazole, 100-200 mg twice daily for 5 days), is the treatment of choice [21]. These drugs are approved but considered experimental for this indication by the FDA. An alternative agent is diethylcarbamazine, a piperazine derivative, $3 \mathrm{mg} / \mathrm{kg}$ divided

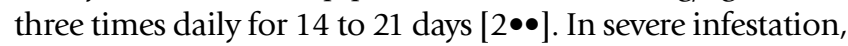
systemic corticosteroids have been advocated to reduce inflammatory complications [23,25].

\section{Capillaria hepatica}

Capillaria hepatica infection is a rare cause of human liver

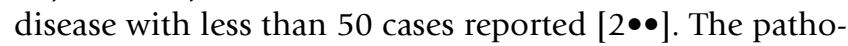
genesis of disease is related to the host immune response as the larvae or eggs produce an eosinophilic granulomatous reaction in the portal tracts $[2 \bullet \bullet, 26]$. Acute hepatitis with hepatic failure has also been reported in a 2 -year-old child [27]. A definitive diagnosis is made by finding of $C$. hepatica on percutaneous liver biopsy. Mebendazole, $200 \mathrm{mg}$ twice daily for 20 days, or albendazole, $400 \mathrm{mg}$ twice daily for 10 days, are two options that may prove efficacious $[13,21 \bullet \bullet]$.

\section{Cestodes (Tapeworms)}

Echinococcus granulosus and Echinococcus multilocularis Background

Four species of Echinococcus cause human hydatid liver disease: E. granulosus (cystic echinococcosis), E. multilocularis (alveolar echinococcosis), and very rarely, E. vogeli or E. oligoarthrus. E. granulosus is distributed worldwide in agrarian societies, particularly in countries where domestic dogs are used for sheep and cattle herding. E. multilocularis is concentrated in temperate regions of the northern hemisphere where dogs and fox species thrive. Villages in central northwest China have recorded prevalence rates as high as $15 \%$ in mass screening programs [28]. Cystic echinococcosis is the most common hydatid liver infestation; however, alveolar echinococcosis is a far more lethal disease. If left untreated, alveolar echinococcosis is fatal in $90 \%$ of cases within 10 years of initial diagnosis and in $100 \%$ within 15 years [29].

\section{Mechanism of disease}

Human echinococcal infestation begins with ingestion of embryonated eggs from food or soil contaminated with host animal feces. The larvae (oncospheres) hatch in the small intestine, penetrate the mucosal wall, and enter the portal or mesenteric circulation. The larvae mature (vesiculate) into fluid-filled hydatid cysts in the liver parenchyma or lungs over several months $[30 \bullet \bullet]$.

The pathogenesis of hydatid liver disease is due largely to mass effect from slow-growing cystic lesions and invasion of adjacent structures. There is a dense, chronic inflammatory infiltrate with fibrosis and granulomatous reaction in the liver surrounding the hydatid lesions. The host cellular immune response appears to be critical in limiting progressive growth of the cysts. A $\mathrm{CD} 4{ }^{+}$predominant T-cell response is associated with slower or abortive disease progression, whereas a $\mathrm{CD} 8^{+} \mathrm{T}$-cell response is found in chronic active infections [31].

\section{Clinical findings}

Cystic hydatid disease is largely asymptomatic until the lesion either reaches an adequate size to produce a mass effect or ruptures. Approximately $70 \%$ of cystic hydatid disease occurs in the liver, with a predilection for the right lobe [14]. Initial symptoms include low-grade fever and tender hepatomegaly with a mild alkaline phosphatase elevation. If the hydatid lesion slowly encroaches upon the hilum of the liver, patients may develop symptoms of progressive cholestasis, venous outflow obstruction, or noncirrhotic portal hypertension. If the cyst ruptures into the biliary system, patients may present with biliary colic, acute cholangitis, or pancreatitis. Significant peripheral eosinophilia is only seen in patients with cyst rupture or leakage [10•]. Secondary bacterial infection can lead to pyogenic liver abscess formation. In addition, cyst leakage has been associated with immune-mediated disease states, including minimal change glomerulonephritis [32].

Alveolar echinococcal liver disease presents in much the same manner as cystic echinococcosis with progressive symptoms due to mass effect, invasion of adjacent structures, and cyst rupture or leakage. Alveolar hydatid liver disease appears to be more insidious and invasive in its presentation and clinical course and is often mistaken for hepatocellular carcinoma initially.

\section{Diagnosis}

Radiologic imaging and confirmation with serologic testing are the mainstay in the diagnosis of hydatid liver disease. Ultrasonography has a sensitivity of approximately $90 \%$. Hydatid lesions are anechoic, round cystic lesions with mixed internal echoes ("hydatid sand") and septations [33]. Hydatid lesions are often difficult to distinguish from benign cysts, hepatic abscesses, or necrotic neoplasia. Abdominal CT has a sensitivity rate of greater than $95 \%$, offers better overall anatomy and detection of hepatobiliary complications, and may thus be the diagnostic test of choice [34]. MRI has been advocated by some authorities and appears to be equal to CT scan in performance characteristics. Magnetic resonance cholangiopancreatography has not been studied in hydatid liver disease.

In all cases of suspected hydatid liver disease, confirmation with a serologic assay is necessary. Specific IgG ELISA tests have sensitivities as high as $94 \%$, specificities of $99 \%$, and negative predictive values of $93 \%$ to $94 \%$ [35]. In addition, ELISA and Western blot assays are able to distinguish between alveolar and cystic echinococcal disease with very low rates of cross-reactivity [35]. PCR amplification of echinococcal DNA and direct detection via Southern hybridization are available for research 
purposes only $[30 \bullet \bullet, 35]$. If the diagnosis remains in doubt, percutaneous aspiration of a cystic lesion and microscopic evaluation (looking for protoscolices and hooklets) can be performed under ultrasound or CT guidance. Patients undergoing aspiration should be placed on benzimidazole before the procedure.

\section{Therapeutic strategy}

Surgical resection of hydatid cysts is the treatment of choice whenever possible because it affords the best chance for cure $[2 \bullet \bullet, 14,30 \bullet \bullet]$. Whether lesions are resectable depends upon the age and general medical condition of the patient as well as the size and location of the cystic lesion or lesions. Postsurgical therapy should be albenda-

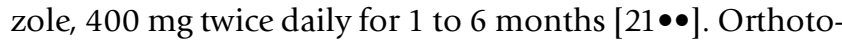
pic liver transplantation has been performed with limited long-term success in Europe [36]. ERCP has also been used successfully in managing biliary complications related to hydatid liver disease before and after surgery [37•].

Percutaneous management of hydatid liver disease is an option for patients who are not surgical candidates. The PAIR (Puncture, Aspiration Injection and Re-aspiration) technique, based on guidelines from the World Health Organization-Informal Working Group on Echinococcosis (WHO-IWGE), is a safe and effective tool in experienced hands $[38 \bullet \bullet$. The recommended adjunctive chemotherapeutic agent is albendazole, $400 \mathrm{mg}$ twice daily, for 15 to 30 days after PAIR treatment depending on the size of the lesion. In alveolar echinococcosis or surgically incurable disease, lifelong benzimidazole therapy is recommended $[21 \bullet \bullet, 30 \bullet \bullet]$. Fluorodeoxyglucose positron emission tomography has been used to monitor metabolic disease activity and guide discontinuation of long-term treatment. However, the majority of patients experience a recurrence within 18 months, and therefore cessation of long-term therapy for alveolar echinococcosis should be discouraged [39].

Disease prevention strategies are now in place in endemic regions of the world. EG95 is a vaccine against $E$. granulosus developed using recombinant DNA techniques for livestock. Two serial vaccinations and a yearly booster have been shown to produce 95\% protection [40]. Massive livestock vaccination programs have shown safety and efficacy in decreasing cystic hydatidosis [41]. The WHO-IWGE has proposed a human vaccine program with a goal of developing the first vaccination against human echinococcosis by 2010 .

\section{Trematodes (Flukes)}

\section{Fasciola hepatica and Fasciola gigantica (liver flukes)} Background

Fasciola hepatica organisms are distributed in temperate, sheep-raising regions of the world such as Central and South America, Europe, Australia, China, and the Middle East. F. gigantica is found in tropical and subtropical areas including southeast Asia, the western Pacific, and Africa. Prevalence estimates range from 2.4 to 17 million cases, with the highest rates reported in Peru and Bolivia [23,42]. The definitive hosts are herbivores, including cattle, sheep, goats, and water buffalo.

\section{Mechanism of disease}

Human Fasciola infestation begins with the ingestion of metacercariae attached to aquatic vegetation, commonly watercress. Transmission of metacercariae has been reported occasionally in contaminated drinking water, and rare cases have been reported from the Middle East of human infestation after consumption of raw animal liver containing larvae [23]. Once in the duodenum, larvae emerge and burrow through the bowel wall into the peritoneal cavity. The larvae penetrate Glisson's capsule and migrate through the hepatic parenchyma to the bile ducts. The larvae mature into adult flukes within the larger intrahepatic bile ducts over 2 to 3 months and begin producing eggs. The eggs are shed in the bile and enter the host intestinal tract through the ampulla of Vater $[2 \bullet \bullet, 14,23]$. To complete the life cycle, the eggs must be shed into fresh water, hatch into miracidia, and enter fresh water snails, where they develop into cercariae.

The pathogenesis of liver disease from fascioliasis is due to the larval migration through the hepatic parenchyma and the chronic inflammatory changes induced within the biliary tract. As the larvae travel through the hepatic parenchyma there is a marked local inflammatory reaction with eosinophilia, hepatocyte necrosis, hemorrhage, and abscess formation followed by fibrosis $[2 \bullet \bullet, 10 \bullet]$. The severity of the hepatic inflammation and damage is proportional to the parasite load; in Bolivia approximately $17 \%$ of cases present with massive infestation [42]. In the chronic biliary phase of infestation, the adult fluke incites hyperplasia and desquamation of the biliary epithelial layer, resulting in thickened, dilated, and fibrotic bile ducts $[2 \bullet \bullet, 14]$. The adult fasciola and its ova, in addition to causing chronic intermittent obstruction, are a nidus for recurrent cholelithiasis. Secondary biliary cirrhosis and sclerosing cholangitis have been reported [23]. The adult fluke is thought to feed on host hepatocytes and biliary epithelial cells while residing in the large intrahepatic and extrahepatic bile ducts [42].

\section{Clinical findings}

The clinical manifestations of fascioliasis are reflective of the life cycle of the parasite. In $50 \%$ of cases, the initial presentation is subclinical [42]. The acute hepatic phase begins within 12 weeks of exposure and is characterized by low-grade fever, tender hepatomegaly, anorexia, nausea, and pruritus. A mild to moderate peripheral eosinophilia is common but can become marked during larval migration through the liver. Elevated levels of alkaline phosphatase and gammaglutamyl transpeptidase are typical, with high aminotransferase levels seen only with signifi- 
cant hepatocellular necrosis [42]. Jaundice, urticaria, weight loss, ascites formation, and hemobilia have been reported in children with massive infestations $[2 \bullet \bullet, 14]$.

During the chronic biliary phase, patients may be asymptomatic or complain of such nonspecific symptoms as dyspepsia, dull right-upper-quadrant discomfort, or diarrhea. If the biliary tract becomes obstructed (either with flukes or gallstones) patients may present with classic biliary colic, ascending cholangitis, acute pancreatitis, or cholecystitis (adult flukes occasionally migrate into the gallbladder). As mentioned previously, chronic inflammation and obstruction have been reported to lead to marked cystic dilation, sclerosing cholangitis, and biliary cirrhosis. In the chronic phase of infestation, peripheral eosinophilia may be mild or absent $[10 \bullet]$.

\section{Diagnosis}

Detection of eggs in the feces, bile, or duodenal aspirate is the definitive test. However, multiple stool specimens and concentration techniques are often necessary in light infestations because egg production and shedding may be low [23]. In a patient with the proper clinical presentation and travel history, a negative stool examination does not rule out fascioliasis. ELISA-based tests aid in diagnosis because of their extremely high sensitivity (>95\%) and specificity $(97 \%-100 \%)[14,23,42]$. Serologic tests are also useful in the acute phase of disease prior to the larvae maturing into adult liver flukes. Serologic titers should decline after successful therapy and therefore can be used to monitor treatment response.

Imaging techniques are also helpful in establishing the diagnosis, particularly in the chronic biliary phase. Hepatic ultrasound may demonstrate adult flukes in the bile ducts or gallbladder as well as hyperechoic lesions corresponding to the burrow tracks of the migrating larvae $[2 \bullet \bullet, 42]$. CT scanning may also reveal characteristic 1 - to $10-\mathrm{mm}$ hypodense linear lesions in a "radiating" pattern that corresponds to the larval burrows in the hepatic parenchyma [42]. In addition, CT may show small peripheral hypodense nodules, calcifications, and a thickened liver

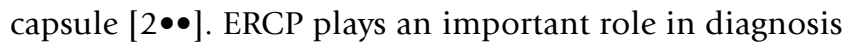
and therapy when biliary obstruction from adult flukes or choledocholithiasis is suspected.

\section{Therapeutic strategy}

The current therapy of choice is triclabendazole, 10 to 12 $\mathrm{mg} / \mathrm{kg} / \mathrm{d}[21 \bullet \bullet]$. However, this drug is not widely available. The alternative therapy is biothionol, 30 to $50 \mathrm{mg} / \mathrm{kg} / \mathrm{d}$ for

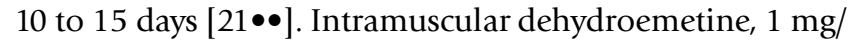
$\mathrm{kg} / \mathrm{d}$ for 14 days, is another alternative [14]. Praziquantel is also used for liver flukes, but because F. hepatica does not respond to this agent it is not recommended $[2 \bullet \bullet, 14,21 \bullet \bullet]$. In biliary obstruction, ERCP with endoscopic sphincterotomy and antihelminthic therapy are recommended. In light infestation, a dormia basket or biliary balloon can be used to clear the bile ducts of adult flukes [42]. In patients with heavy fluke burden and resistance to oral therapy, intraductal endoscopic treatment with a fasciolicidal agent has been successful [43]. A 10\% povidine iodine solution (mixed with contrast) is instilled into the biliary system and left in place by occluding the distal common bile duct with a biliary balloon (for 10 minutes), after which the ducts can be swept free of the dead liver flukes [43].

\section{Clonorchis sinensis, Opisthorchis felineus, and Opisthorcis viverrini (liver flukes) \\ Background}

Clonorchis sinensis is endemic to Hong Kong, southern China, Japan, Korea, and Vietnam. It has been estimated that 7 million people may be infected with Clonorchis $[2 \bullet \bullet, 44]$. Opisthorchis felineus is found mainly in eastern Europe, particularly in Poland, Germany, and Russia [14]. $O$. viverrini is the most common liver fluke infestation in southeast Asia. The World Health Organization estimates that 16 million people worldwide are infested with $O$. felineus and another 10 million with $O$. viverrini. These three liver flukes are found in close association with fisheating mammals and the human consumption of raw or pickled fish. As in fascioliasis, these liver flukes can survive for decades in the host biliary system [44].

\section{Mechanism of disease}

Clonorchis and Opisthorchis species have a similar life cycle to Fasciola species with slightly different definitive and intermediate hosts. The cercariae emerge from fresh water snails and encyst in the muscle of certain fresh water fish species, where they develop into metacercariae. Humans acquire the infestation when they ingest raw, undercooked, or pickled fish that carry the metacercariae. Larvae excyst from the ingested metacercariae in the duodenum or proximal jejunum and migrate into the biliary system through the ampulla of Vater. Once the larvae reach the appropriately sized intrahepatic bile ducts they mature into adult liver flukes over approximately 1 month and begin producing eggs $[23,44]$.

The pathogenesis of liver disease in these fluke infestations is due to the acute and chronic changes induced in the biliary tract. During their migration, the metacercariae incite inflammation, ulceration, and desquamation of the bile duct epithelium. Adenomatous hyperplasia of the bile duct epithelial layer has also been observed [14,44]. Host immune response, bacterial superinfection, and excess mucin production may be responsible for the changes in

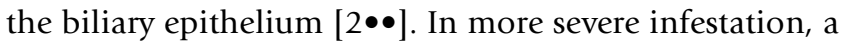
periductular infiltration of eosinophils with necrosis and surrounding hepatocyte atrophy can occur $[2 \bullet \bullet]$; this severe injury results in marked fibrosis and thickening of the bile ducts, referred to as encapsulating fibrosis. In addition, repeated infestation or chronicity can lead to recurrent pyogenic cholangitis as well as secondary biliary cirrhosis or sclerosing cholangitis $[2 \bullet \bullet, 23]$. The development of cholangiocarcinoma is associated with chronic 
infestations of C. sinensis in China and O. viverrini in Thailand $[14,23,44]$.

\section{Clinical findings}

The majority of patients are asymptomatic during the acute infestation. However, up to $10 \%$ of patients present with fever, malaise, dull right-upper-quadrant pain, and mild to moderate peripheral eosinophilia $[10 \bullet, 23]$. Lymphadenopathy and tender hepatomegaly may be detected on physical examination. With obstruction from parasite eggs or recurrent pigment stones, patients may have classic biliary colic, ascending cholangitis, or acute pancreatitis. If the adult flukes migrate into the gallbladder, patients may present with symptoms of acute or chronic cholecystitis. Laboratory evaluation may be suggestive of pancreatobiliary obstruction with or without cholangitis; peripheral eosinophilia is typically absent in chronic liver fluke infestation [10•]. Cholangiocarcinoma should be considered in all patients from endemic regions presenting with weight loss, anorexia, and jaundice.

\section{Diagnosis}

Detection of eggs in the feces, bile, or duodenal aspirate is the definitive test. However, multiple stool specimens and concentration techniques are often necessary in light infestations because egg production may be low $[23,44]$. Serologic tests are available for Clonorchis (immunoblot antigen assay and ELISA) and O. viverrini (ELISA) but are not routinely used except as an adjunct to stool studies or cholangiography $[2 \bullet \bullet, 14,44]$.

Transabdominal ultrasonography detects abnormalities in approximately $50 \%$ of patients with liver fluke infestation. Findings on ultrasound may be consistent with chronic cholecystitis, biliary sludging, hepatomegaly, or hydropic gallbladder. Liver flukes appear as non-shadowing echogenic material within the bile ducts. CT scanning may also reveal dilated or thickened intrahepatic bile ducts that are not appreciated on liver ultrasonography. Cholangiography may reveal duct wall irregularities, multiple cystic dilations of the intrahepatic ducts with tapering at the periphery, and an overall decrease in intrahepatic radicles [44]. If visible, Clonorchis appears as a wavy, elliptiform-filling defect [44].

\section{Therapeutic strategy}

The treatment of choice is praziquantel, $75 \mathrm{mg} / \mathrm{kg}$ divided

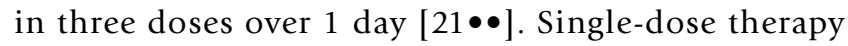
(praziquantel, $40 \mathrm{mg} / \mathrm{kg}$ ) is not advocated in Clonorchis species due to low cure rates. The recommended alternative

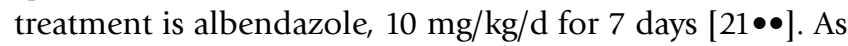
an adjunct to medical therapy, endoscopic management of acute and chronic complications may be necessary. Biliary decompression is often needed in patients with ascending cholangitis on an emergent basis. Chronic complications include recurrent intrahepatic pigment stones, biliary stric- tures, and cholangiocarcinoma and can be managed endoscopically or surgically depending upon regional availability and local expertise.

\section{Schistosoma mansoni, Schistosoma japonicum, Schistosoma intercalatum, and Schistosoma mekongi (blood flukes) \\ Background}

Schistosomiasis affects nearly 200 million people across 77 countries worldwide $[45 \bullet \bullet, 46]$. Schistosoma mansoni (subSaharan Africa, the Middle East, South America, and the Caribbean) and S. japonicum (China, Thailand, Indonesia, and the Philippines) account for the majority of gastrointestinal and hepatosplenic infections $[14,45 \bullet \bullet]$. S. mekongi, found in Laos and Cambodia, may also cause hepatic disease [14]. S. intercalatum infection results in gastrointestinal schistosomiasis but typically does not involve the liver $[45 \bullet \bullet$ ]. Schistosoma species are found in close association with several species of fresh water snails; humans are the definitive host, whereas other vertebrates serve as reservoir hosts [46]. All schistosomal disease occurs within 36 degrees latitude north and south of the equator due to the water temperature requirements of the intermediate host snail [46].

\section{Mechanism of disease}

Human infestation begins when the free-living cercariae penetrate the skin of their host. Once in the subcutaneous tissue, the cercariae become schistosomula and migrate through the venous system to the pulmonary capillaries. The parasites cross the pulmonary vasculature into the left atrium, where the schistosomula are then disseminated to the liver. After 6 to 12 weeks, paired adult male and female worms migrate against portal flow to their respective organs via the inferior or superior mesenteric veins $[14,23,45 \bullet \bullet]$. The eggs reach the terminal venules and penetrate through the vessel walls into the lumen of the intestine, where they are shed in feces. It is estimated that 50\% of ova do not cross into the intestinal lumen and are delivered back to the liver in the venous circulation or are retained in the local tissue [23]. Adult worms can live in the host bloodstream for 30 to 40 years [45••]. The mechanisms by which the adult worms evade host immune detection are unclear but may involve absorption of host proteins and host antigen presentation [23].

The pathogenesis of liver disease in schistosomiasis is directly related to the host immune response to the highly antigenic parasite eggs. Acute schistosomiasis (Katayama fever) is seen in people infected for the first time, typically children or travelers, and is caused by immune-complex deposition in the host tissues $[45 \bullet \bullet, 47]$. Chronic schistosomiasis is due in large part to the vigorous granulomatous response to the parasite ova and the resulting fibrosis. The chronic inflammatory changes in the portal vein radicles (and periportal tissue) result in "pipe stem fibrosis" (also referred to as Symmers' pipe-stem fibrosis). The portal 
fibrosis, along with the granulomatous reaction, leads to presinusoidal portal hypertension $[45 \bullet \bullet, 48]$. This presinusoidal fibrosis does not involve the hepatic parenchyma and therefore is distinct from portal hypertension caused by cirrhosis; liver synthetic function is preserved. However, fibrosis does not occur in everyone with chronic schistosomiasis, and it appears that the genetic composition of the host may play a role [48].

\section{Clinical findings}

Acute schistosomiasis is caused by immune-complex deposition and therefore presents as a serum sickness-like illness. Common clinical findings include fever, myalgia, headache, lymphadenopathy, hematochezia, and right-upper-quadrant pain. Tender hepatomegaly is typical, and in one third

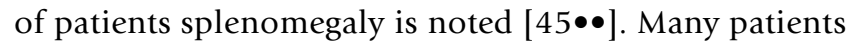
complain of respiratory symptoms, and an interstitial pattern may be seen on chest radiography. A marked peripheral eosinophilia (>3000 eosinophils $/ \mathrm{cm}^{3}$ of blood) is often seen in severe disease, and all patients have at least a mild to moderate response [10•]. If the small bowel is heavily involved, severe hypoproteinemia with edema can occur due to protein-losing enteropathy [14].

The severe portal and periportal fibrosis leading to presinusoidal hypertension occurs in less than $10 \%$ of chronically infected patients $[46,47]$. Hepatomegaly, hypersplenism, refractory thrombocytopenia, gastroesophageal varices, and ascites are long-term complications $[14,45 \bullet \bullet, 46]$. Hepatomegaly is seen in $15 \%$ to $20 \%$ of children during the first year of infestation and is caused by granulomatous lesions in the portal tracts [23]. Hepatic synthetic function and lobular architecture are preserved in pure chronic schistosomiasis. However, viral coinfection with hepatitis B (HBV) or hepatitis C (HCV), as well as alcoholic liver disease, has been associated with a rapid progression to cirrhosis and decompensated liver disease $[45 \bullet \bullet, 49]$. In Egypt, the reported relative risk for hepatocellular carcinoma in chronic $S$. mansoni infection is 5.2 and increases to 12.5 for patients coinfected with HBV, compared with control subjects [49]. Egypt has an exceptionally high prevalence of HCV infection due to the reuse of needles during mass antischistosomal therapy campaigns $[45 \bullet \bullet]$.

\section{Diagnosis}

The definitive diagnosis of schistosomiasis is made with the detection of eggs in the feces or urine of a patient with the appropriate exposure history. The Kato-Katz thick smear stool examination has a reported sensitivity of $90 \%$ when three serial stool samples are submitted $[45 \bullet \bullet, 46]$. Other formalin-based stool sedimentation and concentration techniques may improve diagnostic yields. In symptomatic patients with negative stool studies, a rectal biopsy is recommended [ $45 \bullet \bullet$. Antibody assays are available but should be limited clinically to patients with acute infestation (ie, returning travelers with dermatitis or Katayama fever) $[45 \bullet \bullet, 46]$.
Laboratory results may vary from mild peripheral eosinophilia and alkaline phosphatase elevation to signs of decompensated liver disease and hypersplenism. Liver biopsy may be necessary in patients with coinfection for accurate staging of the severity of parenchymal disease $[45 \bullet \bullet]$. Radiologic imaging can be helpful in measuring the liver and spleen size as well as portal vein flow, splenic vein diameter, and the presence or absence of collaterals. It can also be helpful for hepatocellular cancer screening.

\section{Treatment}

Therapy for schistosomiasis depends upon the stage of disease and clinical presentation. The drug of choice is praziquantel. Although its exact mechanism of action is unknown, praziquantel appears to cause spastic paralysis of the parasite $[23,45 \bullet \bullet$. In S. mansoni and S. haematobium, the recommended dosage is $40 \mathrm{mg} / \mathrm{kg} / \mathrm{d}$ divided in two doses over a single day; in S. japonicum and S. mekongi the dosage is 60

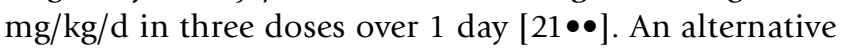
treatment for $S$. mansoni infection is oxamniquine, 15 to 20 $\mathrm{mg} / \mathrm{kg}$ or, in cases arising from Africa, $60 \mathrm{mg} / \mathrm{kg}$ in a single dose [46]. However, oxamniquine is contraindicated in pregnancy $[21 \bullet \bullet]$. Praziquantel and oxamniquine have reported cure rates of $70 \%$ to $90 \%$ and can be given safely to patients with hepatosplenic schistosomiasis $[14,45 \bullet \bullet]$. In acute schistosomiasis, prednisone, 15 to $20 \mathrm{mg} / \mathrm{d}$, has been advocated for 48 to 72 hours before administration of praziquantel $(75 \mathrm{mg} / \mathrm{kg}$ divided in three doses over 1 day) [14]. Chemoprophylaxis with praziquantel is not recommended because of its short half-life and inability to kill schistosomula; however, artemether appears to be a good candidate for mass prophylaxis in endemic regions $[45 \bullet \bullet]$. Vaccine development could aid in the worldwide prevention of schistosomiasis. Phase I and II clinical vaccination trials have shown safety and adequate immune responses to $S$. haematobium-derived proteins with the Sh28GST vaccine (Bilhvax; Developed by Institute Pasteur de Lille, France and produced by Pharos Eurogentec, Belgium) [50].

\section{Conclusions}

Human helminthic infestation is exceedingly common on a global scale, and the liver is frequently the primary organ involved. Helminthic disease manifestations vary from the extremes of asymptomatic carriage to cirrhosis and decompensated liver disease. Stool studies, radiologic imaging, and serologic testing are the mainstays in diagnosis. However, having a high index of suspicion is a critical step in the diagnosis and management of patients with hepatic helminthiasis. Because these parasitic worms can survive for decades in their human host, we must consider all travel history, no matter how remote, significant. Researchers and clinicians alike are moving forward with newer chemoprophylactic and vaccine preventive strategies in an effort to decrease the morbidity and mortality caused by helminthic infestation worldwide. 
References and Recommended Reading Papers of particular interest, published recently, have been highlighted as:

- Of importance

- Of major importance

1. Khuroo MS: Ascariasis. Gastroenterol Clin North Am 1996, 25(3):553-578

2.•• DiazGranados CA, Duffus WA, Albrecht H: Parasitic diseases of the liver. In Hepatology: A Textbook of Liver Disease. Edited by Zakim D, Boyer TD. Philadelphia: WB Saunders; 2002:1073-1107.

An excellent, in-depth review of parasitic infections and their effect on the liver. The chapter includes recent findings on host-parasite immunology and therapeutic management.

3. Sarinas PS, Chitkara RK: Ascariasis and hookworm. Semin Respir Infect 1997, 12:130-137.

4. Thein-Hliang: A profile of ascariasis morbidity in Rangoon Children's Hospital, Burma. J Trop Med Hyg 1987, 90:165-169.

5. World Health Organization:Intestinal protozoan and helminthic infections: report of a WHO scientific group. In Geneva, Switzerland: World Health Organization; 1981. WHO Technical Report Series 666

6. Seltzer E, Barry M: Ascariasis. In Tropical Infectious Diseases: Principles, Pathogens and Practice. Edited by Guerrant RL, Walker DH, Weller PF. Philadelphia: Churchill Livingstone; 1999:959-965.

7. Khuroo MS, Zargar SA, Mahajan R: Hepatobiliary and pancreatic ascariasis in India. Lancet 1990, 335:1503-1506.

8. Schulman A: Intrahepatic biliary stones: imaging features and a possible relationship with Ascaris lumbricoides. Clin Radiol 1993, 47:325-332.

9. Fogaca HS, Oliveira CS, Barbarosa HT, et al: : Liver pseudotumor: a rare manifestation of hepatic granulomata caused by Ascaris lumbricoides ova. Am J Gastroenterol 2000, 95:2099-2101.

10. Ryan ET, Wilson ME, Kain KC: Illness after international travel. N Engl J Med 2002, 347:505-516.

This article reviews the clinical presentations for various parasitic and nonparasitic infections in the traveler. The review includes an excellent table on eosinophilia and associated findings.

11. Sandouk F, Haffar S, Zada MM, et al.: Pancreatic-biliary ascariasis: experience of $\mathbf{3 0 0}$ cases. Am J Gastroenterol 1997, 92:2264-2268.

12. Albonico M, Smith PG, Hall A: A randomized controlled trial comparing mebendazole and albendazole against Ascaris, Trichuris and hookworm infections. Trans $R$ Soc Trop Med Hyg 1994, 88:585-589.

13. Liu LX, Weller PF: Antiparasitic drugs. N Engl J Med 1996, 334:1178-1184.

14. Dunn MA: Parasitic diseases. In Schiff's Diseases of the Liver. Edited by Schiff ER, Sorrell MF, Maddrey WC. Philadelphia: Lippincott Williams \& Wilkins; 2003:1509-1527.

15. Marques de Oliveira LC, Toffoli-Ribeiro C, de Melo Mendes D, et al.: Frequency of Strongyloides stercoralis infection in alcoholics. Mem Inst Oswaldo Cruz 2002, 97:119-121.

16. Parana R, Portugal M, Vitvitski L: Severe strongyloidiasis during interferon plus ribavirin therapy for chronic HCV infection. Eur J Gastroenterol Hepatol 2000, 12:245-246.

This case report is an important consideration due to the increasing burden of HCV and frequency of therapy. The authors conclude that the ribavirin was likely responsible for the clinical presentation.

17. Atkins NS, Conway DJ, Lindo JF, et al.: L3 antigen-specific antibody isotype responses in human strongyloidiasis: correlations with larval output. Parasite Immunol 1999, 21:517-526.

18. Tsai HC, Lee SS, Liu YC, et al.: Clinical manifestations of strongyloidiasis in southern Taiwan. J Microbiol Immunol Infect 2002, 35:29-36.

19. Sato Y, Kobayashi J, Toma H, Shiroma Y: Efficacy of stool examination for detection of Strongyloides infection. Am J Trop Med Hyg 1995, 53:248-250.

20. van der Feltz M, Slee PH, van Hees PA, et al.: Strongyloides stercoralis infection: how to diagnose best? Neth J Med 1999, 55:128-131.
21.• Drugs for parasitic infections. The Medical Letter on Drugs and Therapeutics. http://www.medletter.com/

This is a comprehensive listing of the most recent recommendations in medical therapy for parasitic infections, including adult and pediatric dosage, duration, and alternatives.

22. Hanck C, Hatz C: Treatment of Strongyloides infections [letter]. Gastroenterology 2000, 119:1805.

23. Liu LX, Weller PF, Nutman TB, et al.: Helminthic infections. In Harrison's Principles of Internal Medicine. Edited by Braunwald E, Fauci AS, Kasper DL, et al. New York: McGraw-Hill; 2001:1231-1248.

24. Schantz PM: Toxocariasis. In Hunter's Tropical Medicine and Emerging Infectious Diseases. Edited by Strickland DT. Philadelphia: WB Saunders; 2000:787-790.

25. Rai SK, Uga $S, W u Z$, et al.: Use of polymerase chain reaction in the diagnosis of toxocariasis: an experimental study. Southeast Asian J Trop Med Pub Health 1997, 28:541-544.

26. Govil H, Desai M: Capillaria hepatica parasitism. Indian J Pediatr 1996, 63:698-700.

27. Terrier P, Hack I, Hatz C, et al.: Hepatic capillariasis in a 2year-old boy. J Pediatr Gastroenterol Nutr 1999, 28:338-340

28. Craig PS, Giraudoux P, Shi D, et al:: An epidemiological and ecological study of human alveolar echinococcosis transmission in south Gansu, China. Acta Trop 2000, 77:167-177.

29. Ammann RW, Eckert J: Cestodes. Gastroenterol Clin North Am 1996, 25:655-689.

$30 . \bullet$ Craig PS: Echinococcus multilocularis. Curr Opin Infect Dis 2003, 16:437-444.

An excellent overall review of Echinococcus with in-depth information on the pathogenesis of disease, parasite biology, and host

immune response.

31. Vuitton DA, Bresson-Hadni S, Laroche $\mathrm{L}$, et al.: Cellular immune response in Echinococcus multilocularis infection in humans II. Natural killer cell activity and cell subpopulations in the blood and in the periparasitic granuloma of patients with alveolar echinococcosis. Clin Exp Immunol 1989, 78:67-74.

32. Gelman R, Brook G, Green J, et al.: Minimal change glomerulonephritis associated with hydatid disease. Clin Nephrol 2000, 53:152-155.

33. Suwan Z: Sonographic findings in hydatid disease of the liver: comparison with other imaging methods. Ann Trop Med Parasitol 1995, 89:261-269.

34. Kervancioglu R, Bayram M, Elbeyli L: CT findings in pulmonary hydatid disease. Acta Radiol 1999, 40:510-514.

35. Silas-Lucas M, Gottstein B: Molecular tools for the diagnosis of cystic and alveolar echinococcosis. Trop Med Int Health 2001, 6:463-475.

36. Bresson-Hadni S, Koch S, Beurton I, et al: Primary disease recurrence after liver transplantation for alveolar echinococcis: long-term evaluation of 15 patients. Hepatology 1999, 30:857-864.

37. Simsek H, Ozaslan E, Sayek I, et al.: Diagnostic and therapeutic ERCP in hepatic hydatid disease. Gastrointest Endosc 2003, 58:384-389.

This retrospective study reviews the endoscopic management of 39 patients with hydatid liver disease, focusing on ERCP findings and the role of ERCP in pre- and postoperative management.

38. $\bullet$ World Health Organization: Communicable Disease Surveillance and Response: PAIR: Puncture, Aspiration, Injection, Re-Aspiration. http://www.who.int/emc-documents/zoonoses/ whocdscsraph20016.html. Accessed March 2004.

A step-by-step analysis of how to perform PAIR therapy.

39. Reuter S, Buck A, Burkhard M, et al.: Structured treatment interruption in patients with alveolar echinococcosis. Hepatology 2004, 39:509-517.

40. Echinonet, on line version of Echinonews: Newsletter of the WHO Informal Working Group on Echinococcosis. http:// www.medicalweb.it/aumi/echinonet Accessed March 2004.

41. Heath DD, Jensen O, Lightowlers MW: Progress in control of hydatidosis using vaccination: a review of formulation and delivery of the vaccine and recommendations for the practical use in control programmes. Acta Trop 2003, 85:133-143. 
42. Roig GV: Hepatic fascioliasis in the Americas: a new challenge for therapeutic endoscopy. Gastrointest Endosc 2002, 56:315-317.

43. Dowidar N, El Sayad M, Osman M, Salem A: Endoscopic therapy of fascioliasis resistant to oral therapy. Gastrointest Endosc 1999, 50:345-351

44. Bunnag D, Cross JH, Bunnag T: Liver fluke infections. In Hunter's Tropical Medicine and Emerging Infectious Diseases. Edited by Strickland DT. Philadelphia: WB Saunders; 2000:840-846.

45.• Ross AG, Bartley PB, Sleigh AC, et al.: Schistosomiasis. N Engl J Med 2002, 346:1212-1220.

This review article is the best in-depth analysis of the pathophysiology, diagnosis, and treatment of schistosomiasis. The authors also review recent vaccine developments.
46. Bica I, Hamer DH, Stadecker MJ: Hepatic schistosomiasis. Infect Dis Clin North Am 2000, 14:583-604.

47. Strickland DT, Ramirez BL: Schistosomiasis. In Hunter's Tropical Medicine and Emerging Infectious Diseases. Edited by Strickland DT. Philadelphia: WB Saunders; 2000:804-832.

48. Sharma S: Granulomatous diseases of the liver. In Hepatology: A Textbook of Liver Disease. Edited by Zakim D, Boyer TD, Walton RE. Philadelphia: WB Saunders; 2002:1317-1330.

49. Stanton RE: Developments in schistosomiasis research and management. Curr Opin Infect Dis 2000, 13:445-450.

50. Capron A, Capron M, Dombrowicz D, Riveau G: Vaccine strategies against schistosomiasis: from concepts to clinical trials. Int Arch Allergy Immunol 2001, 124:9-15. 